\title{
ON ABELIAN QUOTIENTS OF PRIMITIVE GROUPS
}

\author{
MICHAEL ASCHBACHER AND ROBERT M. GURALNICK
}

(Communicated by Warren J. Wong)

\begin{abstract}
It is shown that if $G$ is a primitive permutation group on a set of size $n$, then any abelian quotient of $G$ has order at most $n$. This was motivated by a question in Galois theory. The field theoretic interpretation of the result is that if $M / K$ is a minimal extension and $L / K$ is an abelian extension contained in the normal closure of $M$, then the degree of $L / K$ is at most the degree of $M / K$.
\end{abstract}

\section{INTRODUCTION}

In this note, we prove the following results:

Theorem 1. Let $G$ be a primitive permutation group on a set of finite order $n$. Then $\left|G: G^{\prime}\right| \leq n$.

Theorem 2. Let $G$ be a permutation group on a finite set of order $n$. Then $\left|G: G^{\prime}\right| \leq 3^{n / 3} \leq 2^{n-1}$.

Theorem 3. Let $V$ be a finite dimensional vector space over a finite field of characteristic $\ell$ and $G$ a subgroup of $\mathrm{GL}(V)$. If $O_{\ell}(G)=1$, then $\left|G: G^{\prime}\right|<|V|$.

Theorem 1 answers a question of Tamagawa, which was prompted by [I]. A minor modification of the argument in [I] shows that $\left|G: G^{\prime}\left(H \cap H^{g}\right)\right| \leq|G: H|$. This was first observed by $\mathrm{D}$. Cantor in the following form: If $\alpha$ and $\beta$ are conjugate over a field $K$, then any abelian extension $L / K$ with $L \subset K(\alpha, \beta)$ satisfies $|L: K| \leq|K(\alpha): K|$. The field theoretic version of Theorem 1 is:

Corollary 4. Let $K$ be a field with $M / K$ a minimal extension. If $L / K$ is an abelian extension with $L$ contained in the normal closure of $M$, then $|L: K| \leq|M: K|$.

Theorems 2 and 3 are needed to prove Theorem 1. Theorem 3 depends on the classification of simple groups (one needs to know the relative sizes of outer

Received by the editors November 10, 1988 and, in revised form, February 8, 1989.

1980 Mathematics Subject Classification (1985 Revision). Primary 20B15; Secondary 20C20, $20 \mathrm{~B} 05$.

Both authors were partially supported by NSF grants. The first author was partially supported by NSA. 
automorphism groups, degrees of permutation representations, and dimensions of modules). See also [KP] in regard to Theorem 2.

It is not very difficult to see that primitive cannot be replaced by transitive in Theorem 1. We shall give an example to show that the bound in Theorem 2 is not too far off even in the transitive case.

\section{TRANSITIVE GROUPS}

(2.1). Let $G$ be a group, $\pi: G \rightarrow A$ a homomorphism, and $K=\operatorname{ker} \pi$. Suppose $H=M \leq G$. Then

$$
|H:[M, H]|=|H \pi:[M, H] \pi||H \cap K:[M, H] \cap K| .
$$

Proof. First $[M \pi, H \pi]=[M, H] \pi \cong[M, H] K / K$. Therefore, $H \pi /[M \pi, H \pi]$ $\cong H K /[M, H] K \cong H /(H \cap[M, H] K)=H /[M, H](H \cap K)$. Then as $|H:[M, H]|$ $=|H:(H \cap K)[M, H]||(H \cap K)[M, H]:[M, H]|$, it remains only to observe that $(H \cap K)[M, H] /[M, H] \cong(H \cap K) /[M, H] \cap K)$.

(2.2). Let $\pi: G \rightarrow A$ be a surjective group homomorphism and $K=\operatorname{Ker} \pi$. Then $\left|G: G^{\prime}\right|=\left|A: A^{\prime}\right|\left|K \cap G^{\prime}\right| \leq\left|A: A^{\prime}\right|\left|K: K^{\prime}\right|$.

Proof. Apply (2.1) with $G=M=H$.

Theorem 2.3. Let $G$ be a group of permutations on a set $X$ of order $n$. Then

(a) $\left|G: G^{\prime}\right| \leq 3^{n / 3} \leq 2^{n-1}$, and

(b) $\left|G: G^{\prime}\right| \leq p^{n / p}$ for $G$ a p-group.

The proof is by induction on $|G|$. So assume $G$ is a minimal counterexample to $(\mathrm{a})$ or $(\mathrm{b})$.

(2.4). $G$ is nilpotent.

Proof. If not, choose $M$ maximal in $G$ with $G=M G^{\prime}$. Then $\left|G: G^{\prime}\right|=$ $\left|M: M \cap G^{\prime}\right| \leq\left|M: M^{\prime}\right| \leq 3^{n / 3}$ by induction.

(2.5). $G$ is transitive on $X$.

Proof. If $G$ acts on a proper subset $Y$ of $X$ with $0 \neq m=|Y|<n$, then apply (2.2) with $\pi$ the representation of $G$ on $Y$ to obtain $\left|G: G^{\prime}\right| \leq 3^{m / 3} 3^{(n-m) / 3}=$ $3^{n / 3}$. Similarly, if $G$ is a $p$-group, we see that $G$ is transitive.

(2.6). $G$ is a p-group for some prime $p$.

Proof. If not then $G=P \times Q$ where $P$ is a $p$-group and $Q$ is a $p^{\prime}$-group. Let $H=G_{x}$ be the stabilizer of $x$ in $X$. Then $H=M \times N$ with $M=H \cap P$ and $N=H \cap Q$. Note that $P$ acts faithfully on the cosets of $M$ as does $Q$ on the cosets of $N$. So

$$
\left|G: G^{\prime}\right|=\left|P: P^{\prime}\right|\left|Q: Q^{\prime}\right| \leq 3^{a / 3} 3^{b / 3} \leq 3^{n / 3},
$$

$$
\text { where } a=|P: M| \text { and } b=|Q: N| \text {. }
$$

Proof of Theorem 2.3. By (2.6) and the fact that $p^{n / p} \leq 3^{n / 3}$ for $p$ a prime, it suffices to prove (2.3b). So $G$ is a transitive $p$-group. Let $Z$ be a central 
subgroup of order $p$. If $G=Z$, then $n=p=\left|G: G^{\prime}\right|$. Otherwise $G$ acts transitively on the set $Y$ of orbits of $Z$. Note $|Y|=n / p$. Let $\pi$ be the representation of $G$ as a permutation group on these orbits. By (2.2) and induction, $\left|G: G^{\prime}\right| \leq p^{n / p^{2}}\left|K: K \cap G^{\prime}\right|$, where $K=\operatorname{Ker} \pi$. Choose $g \in G$ with no fixed points on $Y$. Then $\left|K: K \cap G^{\prime}\right| \leq|K:[g, K]| \leq\left|C_{K}(g)\right| \leq p^{n / p^{2}}$. Hence $\left|G: G^{\prime}\right| \leq p^{2 n / p^{2}} \leq p^{n / p}$.

By considering elementary abelian 3-subgroups of $S_{n}$, we see that $(2.3 \mathrm{~b})$ cannot be improved.

We use the classification of finite simple groups in the following form:

Lemma 2.7. Let $L$ be a finite nonabelian simple group and $M$ a proper subgroup of $L$ with $|L: M|=k$.

(i) If $L \neq A_{6}$, then $2 \mid$ Out $L \mid<k$.

(ii) $4 \mid$ Out $L|<| L \mid$.

(iii) If $L \leq \operatorname{PSL}_{d}(q)$, then $2 \mid$ Out $L \mid<q^{d} /(q-1)$.

Proof. This follows easily by inspection. Note (ii) and (iii) follow from (i).

Proposition 2.8. Let $G$ be a primitive permutation group of degree $n$ on a set $X$. Then either

(i) $\left|G: G^{\prime}\right|<n / 2$, or

(ii) $G$ preserves an affine structure on $X$.

Proof. Assume (2) does not hold. First consider the case where $F^{*}(G)=D=$ $L_{1} \times \cdots \times L_{r}$ is the product of $r$ nonabelian simple groups $L_{i}$ permuted transitively by $G$. It follows by [AS, Theorem 1] that either $n=k^{r}$ for $k=\left|L_{1}: M\right|$ for some proper subgroup $M$ of $L_{1}$ or that $n=\ell^{s} \geq \ell^{r / 2}$ where $\ell=\left|L_{1}\right|$ and $r \geq 2$. Let $K=\bigcap_{i} N_{G}\left(L_{i}\right)$. By Theorem 2, $\left|G: G^{\prime} K\right| \leq 2^{r-1}$. Moreover, $K / D \leq$ Out $L_{1} \times \cdots \times$ Out $L_{r}$. If $r=1$, then $\left|G: G^{\prime}\right| \leq \mid$ Out $L_{1} \mid<k / 2$ by 2.7(i) unless $L_{1}=A_{6}$. If $L_{1}=A_{6}$, then either $k=6$ and $\left|G: G^{\prime}\right| \leq 2$ or $k>8$ and $\left[G: G^{\prime}\right] \leq 4$. If $r>1$, then (3.1) implies $|K / D:[G, K / D]| \leq \mid$ Out $\left.L_{1}\right|^{r / 2}$. Thus $\left|G: G^{\prime}\right|=\left|G: G^{\prime} K \| K: K \cap G^{\prime}\right| \leq 2^{r-1} \mid$ Out $\left.L_{1}\right|^{r / 2}$. If $n=k^{r}$, then $2.7(\mathrm{i})$ implies $\left|G: G^{\prime}\right|<n / 2$. If $n=\ell^{s}, s \leq r / 2$, then $\left|G: G^{\prime}\right| \leq(4 \mid \text { Out } L \mid)^{r / 2} / 2<n / 2$ by 2.7 (ii).

By [AS], the only case left is when $F^{*}(G)=Q=R \times D$, where $R \cong D$ and $D$ is as above. Moreover, if $H$ is a point stabilizer, then $G=H D$ and $H \cap Q=\{(x, \lambda(x)) \mid x \in R\}$ for some isomorphism $\lambda$ of $R$ and $D$. In particular, $n=|D|=\ell^{r}$ where $l=\left|L_{1}\right|$. Arguing as above, we see that $\left|G: G^{\prime}\right| \leq$ $\ell^{r / 2} / 2<n / 2$. 


\section{INDUCED MODULES}

(3.1). Let $X=X_{1} \times \cdots \times X_{m}, m>1$, be the direct product of finite groups $X_{i}$ permuted transitively by $G$. If $V$ is a $G$-invariant subgroup of $X$, then $|V:[G, V]| \leq|X|^{1 / 2}$.

Proof. Choose $g \in G$ that leaves no $X_{i}$ invariant. Then $\left|C_{X}(g)\right| \leq|X|^{1 / 2}$. Hence $|V:[G, V]| \leq|V:[g, V]| \leq\left|C_{V}(g)\right| \leq\left|C_{X}(g)\right| \leq|X|^{1 / 2}$.

(3.2). Let $F$ be a finite field of order $q$ and $G$ the group of maps $\tau(a, \sigma): x \rightarrow$ $a(x \sigma)$ on $F$, where $a \in F^{\#}$ and $\sigma \in$ Aut $F$. Let $B=\left\{\tau(a, 1) \mid a \in F^{\#}\right\}$ and $H \leq G$. Then either

(1) $\left|H: H^{\prime}\right| \leq q / 2$, or

(2) $H=B$ has order $q-1$.

Proof. If $H \leq B$, the result is clear. So assume not. Then $e=|H: H \cap B|>1$. Hence $q=r^{e}$ and $H=\langle\alpha, h\rangle$, where $\langle h\rangle=H \cap B$ and $\alpha(x)=a x^{r}$ for some $a \in F^{\#}$. Then $\left|C_{B}(\alpha)\right|=r-1$. Thus $\left|H: H^{\prime}\right|=|H: H \cap B|\left|H \cap B: H^{\prime}\right|=$ $e\left|C_{H \cap B}(\alpha)\right| \leq e(r-1) \leq q / 2$.

\section{LINEAR ACTIONS}

In this section $F$ is a finite field of characteristic $l, V$ is a finite dimensional vector space over $F$, and $G \leq \mathrm{GL}(V)$. We prove:

Theorem 4.1. Assume $O_{\ell}(G)=1$. Then either:

(a) $\left|G: G^{\prime}\right| \leq|V| / 2$, or

(b) $G$ is abelian and $|G|<|V|$.

Let $(G, V)$ be a minimal counterexample to (4.1). Clearly $G$ is nonabelian. (4.2). $G$ acts irreducibly on $V$.

Proof. If not, choose $0=V_{0}<V_{1}<\cdots<V_{d}=V$ with $V_{i} G$-invariant and $W_{i}=V_{i} / V_{i-1} G$-irreducible. Since $O_{\ell}(G)=1, G$ acts faithfully on $W=W_{1} \oplus \cdots \oplus W_{d}$. So we can assume $V$ is a semisimple module. Since $G$ is nonabelian, there exists an irreducible submodule $U$ such that $\pi(G)$ is nonabelian, where $\pi$ is the representation of $G$ on $U$. Thus if $K=\operatorname{ker} \pi$, $\left|G: G^{\prime} K\right|<|U| / 2$. Since $O_{\ell}(K)=1, K$ acts faithfully on $V / U$. So by minimality, $\left|K: K^{\prime}\right| \leq|V / U|$, whence $\left|G: G^{\prime}\right| \leq|V| / 2$.

(4.3). $G$ is primitive on $V$.

Proof. If not, there is a nontrivial decomposition $V=\bigoplus_{i} V_{i}$ with $G$ permuting $X=\left\{V_{1}, \ldots, V_{r}\right\}$ and $N_{G}\left(V_{1}\right)$ acting primitively on $V_{1}$. Let $\pi$ be the permutation representation of $G$ on $X$ and $K=\operatorname{ker} \pi$. Set $K_{0}=K$ and $K_{i+1}=\left\{g \in K_{i} \mid g\right.$ acts trivially on $\left.V_{i+1}\right\}$. By (2.2), $\left|G: G^{\prime}\right|=k_{0} k_{1} \ldots k_{r}$, where $k_{0}=\left|G: G^{\prime} K\right|$ and $k_{i}=\left|K_{i-1}:\left(K_{i-1} \cap G^{\prime}\right) K_{i}\right| \leq\left|K_{i-1}: K_{i-1}^{\prime} K_{i}\right|$ for $i \geq 1$. By Theorem $2, k_{0} \leq 2^{r-1}$. 
Since $N_{G}\left(V_{i}\right)$ acts primitively on $V_{i}$ and $K \unlhd N_{G}\left(V_{i}\right), K$ acts homogeneously on each $V_{i}$. If $K$ is not irreducible on $V_{1}$ (and so not on each $V_{i}$ ), then $k_{i} \leq\left|K_{i-1}: K_{i-1}^{\prime}\right|<|W| \leq\left|V_{i}\right| / 2$, where $W$ is a $K$-irreducible subspace of $V_{1}$. Thus $\left|G: G^{\prime}\right|<2^{r-1}(|V| / 2)^{r}=|V| / 2$.

So assume $K$ acts irreducibly on $V_{i}$. If $K_{i-1} / K_{i}$ is not abelian for each $i$, then $k_{i} \leq\left|K_{i-1}: K_{i-1}^{\prime} K_{i}\right| \leq\left|V_{i}\right| / 2$ by induction, and $\left|G: G^{\prime}\right| \leq|V| / 2$ as above. So we can assume some $K_{i-1} / K_{i}$ is abelian. Then $\left|K_{i-1} / K_{i}\right|<\left|V_{i}\right|$, whence $\left|K_{i-1}:\left(K_{i-1} \cap G^{\prime}\right) K_{i}\right| \leq\left|V_{i}\right| / 2$ unless $K_{i-1} \cap G^{\prime} \leq K_{i}$. Since $K$ acts irreducibly on $V_{i}$ and $K_{i-1} \unlhd K$, this can happen only if $K_{i-1} / K_{i}$ is cyclic of order $\left|V_{i}\right|-1$ and $\left[K, K_{i-1}\right] \leq K_{i}$, whence $K$ is abelian. If $K$ is abelian, then $|K| \leq\left(\left|V_{1}\right|-1\right)^{r}$, and so by (3.1), $|K:[G, K]| \leq|K|^{1 / 2}$. Thus $\left|G: G^{\prime}\right| \leq$ $2^{r-1}\left(\left|V_{1}\right|-1\right)^{r / 2}<|V| / 2$.

(4.4). If $D \unlhd G$, then $D$ acts homogeneously. In particular, if $D$ is abelian, then $D$ is cyclic.

Proof. Apply (4.3).

(4.5). If $D$ is a noncyclic normal subgroup of $G$, then $D$ acts irreducibly on $V$ and $C_{G}(D)$ is cyclic.

Proof. By (4.4), $D$ acts homogeneously. So assume $V=V_{1} \oplus \cdots \oplus V_{m}, V_{i} \cong V_{j}$ as irreducible $D$-modules. Let $E=$ End $_{F D} V_{1}$ with $q=|E|$ and $m>1$. Set $U=V_{1}$. Let $\alpha: D \rightarrow \mathrm{GL}(U)$ be the representation of $D$ on $U$. Let $\Gamma$ be the normalizer of $D \alpha$ in $\operatorname{GL}(U)$ and $S$ the centralizer of $D \alpha$. Since $g V_{1} \cong V_{1}$ as $F D$-modules for each $g \in G$, we can define $\pi: G \rightarrow \Gamma / S$ by $g \pi=S x$ where $\alpha\left(g^{-1} h g\right)=x^{-1} \alpha(h) x$. Note $K=\operatorname{ker} \pi=C_{G}(D)$. Let $M$ be the inverse image of $G \pi$ in $\Gamma \leq \mathrm{GL}(U)$. Since $M$ contains a copy of $D, M$ is nonabelian and so by minimality, $\left|G: G^{\prime} K\right| \leq\left|M: M^{\prime}\right| \leq|U| / 2$. Since $K=C_{G}(D)$, there exists a faithful representation $\beta: K \rightarrow \mathrm{GL}_{m}(E)$. By minimality, $\left|K: K^{\prime}\right|<q^{m}$. Thus $\left|G: G^{\prime}\right|<|U| q^{m} / 2 \leq|U| q^{2 m-2} / 2 \leq|U|^{m} / 2=|V| / 2$.

Let $T$ be a maximal normal cyclic subgroup of $G$.

(4.6). $T \neq C_{G}(T)$.

Proof. If so, then (3.2) applies.

Now choose $D$ minimal subject to $D \leq C_{G}(T), D \unlhd G$, and $D$ is nonabelian. Thus by (4.4), (4.5), and the maximality of $T$ :

(4.7). $Z(D)$ is the largest characteristic abelian subgroup of $D, Z(D)$ is cyclic, $D$ acts irreducibly on $V$, and $T=C_{G}(D)$.

(4.8). Either

(1) $D$ is the central product of quasisimple subgroups $L_{1}, \ldots, L_{r}$ permuted transitively by $G$, or 
(2) $D=P Z(D)$ with $|P|=p^{1+2 w}$ and $P$ an extraspecial $p$-group and either $Z(D)=Z(P)$ or $p=2$ and $Z(D) \cong Z_{4}$. Moreover, $G$ is irreducible on $\tilde{D}=D / Z(D)$.

Proof. This follows from (4.7) and the minimal choice of $D$.

(4.9). (4.8.2) holds.

Proof. Assume (4.8.1) holds. Let $X=\left\{L_{1}, \ldots, L_{r}\right\}, \pi$ the permutation representation of $G$ on $X$ and $K=\operatorname{ker} \pi$. By (4.5), $D$ acts irreducibly on $V$. Let $E=\operatorname{End}_{D} V$ and set $q=|E|$. Since $V$ is absolutely irreducible over $E$, $V=V_{1} \otimes_{E} \cdots \otimes_{E} V_{r}$ where $V_{i}$ is an absolutely irreducible $\hat{L}_{i}$-module (where $\hat{L}_{i}$ is the covering group of $\left.L_{i}\right)$. Let $d=\operatorname{dim}_{E} V_{i}$. Then $|V|=q^{d^{r}}$ and End $_{L_{1}} V_{1}=E$. By Lemma $2.7($ iii) $) 2 k<q^{d} /(q-1)$, where $k=\mid$ Out $L_{1} \mid$. In particular, $(2 k)^{r}(q-1)<q^{d^{r}}=|V|$. Since $T=C_{G}(D)$ by (4.7), it follows that $|T|<q$ and that $\left|K: K^{\prime}(T \cap K)\right| \leq k^{r}$. By Theorem 2, $\left|G: G^{\prime} K\right| \leq 2^{r-1}$. Thus

$$
\left|G: G^{\prime}\right| \leq 2^{r-1} k^{r}(q-1)=(2 k)^{r}(q-1) / 2<|V| / 2 .
$$

(4.10). Let $q=\left|\operatorname{End}_{D} V\right|$. Then $q>\left|C_{G}(D)\right|,|V| \geq q^{p^{\prime \prime}}$, and $q \equiv 1 \bmod p$.

Proof. Clearly $q>|T|$. Since $V$ is a faithful $D$-module, the second inequality holds. Since $\operatorname{Aut}_{P} V \geq Z(D), q \equiv 1 \bmod p$.

(4.11). $C_{G}(\tilde{D})=D C_{G}(D)$.

Proof. Any automorphism of $D$ which is trivial on $\tilde{D}$ must be inner.

Proof of Theorem 4.1. Now $G$ acts irreducibly on $\tilde{D}$. Since $D \leq G^{\prime} Z(D)$, it follows by the minimality of $(G, V)$ that $\left|G: G^{\prime} C_{G}(\tilde{D})\right|<|\tilde{D}|$. Thus $\left|G: G^{\prime}\right|<$ $\left|\tilde{D} \| C_{G}(D): C_{G}(D) \cap G^{\prime}\right| \leq p^{2 w \prime}(q-1) / p<q^{p^{\prime \prime}} / 2 \leq|V| / 2$ unless $q=3, p=2$, $w=1$, and $|V|=9$. The result follows by inspection in this case.

\section{Primitive Groups}

In this section we prove the following result:

Theorem 1. Let $G$ be a primitive group of permutations on a set $X$ of order $n$. Then $\left|G: G^{\prime}\right| \leq n$.

The proof is by induction on $n$. So choose a counterexample with $n$ minimal. By (2.7):

(5.1). G preserves an affine space structure on $X$.

By (5.1) $F^{*}(G)=V$ is an $m$-dimensional vector space over $F=\mathrm{GF}(p)$ for some prime $p$, with $V$ regular on $X$ and $G=H V$, where $H=G_{X}$ is the stabilizer of some $x \in X$ and $H$ is an irreducible subgroup of $\operatorname{GL}(V)$. Then $n=|V|$. We can assume $H \neq 1$. Also as $H$ is irreducible on $V$, $V=[V, H] \leq G^{\prime}$. So $\left|G: G^{\prime}\right|=\left|H: H^{\prime}\right|$. Finally by Theorem $3,\left|H: H^{\prime}\right|<|V|$. This contradiction completes the proof of Theorem 1. 
Note that in fact one has the slightly stronger result that either $\left|G: G^{\prime}\right| \leq n / 2$ or $G^{\prime}$ is abelian and $G / G^{\prime}$ is cyclic.

\section{TRANSITIVE SUBgROUPS II}

We would like to know whether the bound in Theorem 2.3 is a good one for transitive subgroups. Observe that the proof of (2.3) actually shows:

(6.1). If $P$ is a p-group acting transitively on a set $X$ of size $p^{a}, a \geq 3$, then $\left|P: P^{\prime}\right| \leq p^{g(a)}$, where $g(a)=2+p+p^{2}+\cdots+p^{a-2}=1+\left(p^{a-1}-1\right) /(p-1)$.

Thus for $p$ large, $\left|P: P^{\prime}\right|$ cannot be much larger than $p^{n / p^{2}}$. So we shall look for a 2-group for an example.

Let $E$ be an elementary abelian group of order $2^{d}$. Let $X=\mathbf{Z}_{2}[E]$ as an $E$-module. Then $X$ has a filtration $X=X_{0} \geq X_{1} \geq X_{2} \geq \cdots \geq X_{d}=0$, such that $(g-1) X_{i} \leq X_{i+1}$ for all $g \in E$. Moreover, $\operatorname{dim} X_{i} / X_{i-1}=\left(\begin{array}{c}d \\ i\end{array}\right)$. For convenience let us assume $d=2 f$ is even.

Set $V=X_{f}$ and $G=V E$. Note that $G \leq X E \cong \mathbf{Z}_{2} w r E \leq S_{n}, n=2^{d+1}$, and that $G$ acts transitively. Also

$$
\left|G: G^{\prime}\right|=|G: V||V:[G, V]|=2^{d+\left(\begin{array}{l}
d \\
f
\end{array}\right)} .
$$

Since $\left(\begin{array}{l}d \\ f\end{array}\right)$ is the largest of the binomial coefficients, it is clear that $\left(\begin{array}{l}d \\ f\end{array}\right) \geq$ $2^{d} /(d+1)=n /\left(2 \log _{2} n\right)$. Thus we have shown:

(6.2). If $n$ is an odd power of 2 , there exists a transitive 2-group $G$ of degree $n$ such that $\left|G: G^{\prime}\right| \geq 2^{n / 2 \log _{2} n}$

If one uses a better approximation for the binomial coefficient, the $\log _{2} n$ can be replaced by $\left(\log _{2} n\right)^{1 / 2}$ for $n$ sufficiently large. See also [A] and [KN] for similar examples.

\section{REFERENCES}

[A] M. S. Audu, Transitive permutation groups of prime-power order, Ph. D. Thesis, Oxford, 1983. [AS] M. Aschbacher and L. Scott, Maximal subgroups of finite groups, J. Algebra 92 (1985), 44-80.

[KN] L. G. Kovacs and M. F. Newman, Generating transitive permutation groups, Quart J. Math Oxford (2) 39 (1988), 361-372.

[KP] L. Kovacs and C. Praeger, Finite permutation groups with large abelian quotients, Pacific J. Math., 136 (1989), 283-292.

[I] I. M. Isaacs, Solution of problem 6523, Amer. Math. Monthly 95 (1988), 561-562.

Department of Mathematics, California institute of Technology, Pasadena, CALIFORNIA 91125

Department of Mathematics, University of Southern California, los Angeles, CALIFORNIA 90089 